http://dx.doi.org/10.32929/2446-8355.2018v27n4p482-492

\title{
CRESCIMENTO DE MUDAS DE SERINGUEIRA (Hevea brasiliensis) EM DIFERENTES TIPOS DE SUBSTRATOS E RECIPIENTES
}

\author{
Jociela Gomes Neres Miranda ${ }^{1}$, Manoel Euzébio de Souza ${ }^{2 *}$, Ana Heloisa Maia ${ }^{2}$ \\ ${ }^{1}$ Graduanda em Agronomia, Universidade do Estado de Mato Grosso-UNEMAT, Câmpus de Nova Xavantina- \\ MT. \\ ${ }^{2}$ Docente do Curso Agronomia, Universidade do Estado de Mato Grosso (UNEMAT), Faculdade de Ciências \\ Agrárias, Biológicas e Sociais Aplicadas, Câmpus de Nova Xavantina-MT.*E-mail: m.euzebio@unemat.br
}

Recebido: 18/02/2018; Aceito: 14/12/18

RESUMO: A seringueira (Hevea brasiliensis) originária da Amazônia tem como principal finalidade a produção de borracha para a fabricação de pneumáticos e diversos produtos importantes à sociedade. Devido a sua rusticidade, esta espécie se adapta a diferentes regiões brasileiras. Um dos aspectos importantes para o estabelecimento dos cultivos comerciais de seringueira envolve a qualidade de mudas, sendo o substrato aliado ao tamanho do recipiente um dos principais fatores para o desenvolvimento satisfatório da muda. Neste contexto, este trabalho teve como objetivo avaliar o desenvolvimento de mudas de seringueira cultivadas em diferentes substratos e tamanhos de recipientes. Os substratos utilizados foram Carolina Padrão comercial (testemunha); esterco bovino + bagaço de cana (1:1); húmus + bagaço de cana (1:1) e húmus e os recipientes foram sacos plásticos de polietileno nas dimensões de 25 x $35 \mathrm{~cm}, 18$ x $32 \mathrm{~cm}$ e 20 x $30 \mathrm{~cm}$, com delineamento experimental utilizado foi em blocos casualizados (DBC) em esquema fatorial 3x4, sendo os fatores analisados três recipientes (sacos plásticos com diferentes dimensões), quatro tipos de substratos e um clone de seringueira, com cinco repetições. A partir dos resultados obtidos no presente trabalho podese concluir que o substrato comercial é o mais indicado para o desenvolvimento inicial de mudas de seringueira, nas condições do experimento. $\mathrm{O}$ mesmo combinado ao recipiente (saco de polietileno tamanho $20 \times 30 \mathrm{~cm}$ ) permitiu um maior comprimento de raízes, influenciando o estabelecimento de plântulas em condições de viveiro.

Palavras-chave: Desenvolvimento. Hevea brasiliensis. Propagação.

\section{GROWTH OF RUBBER TREE (Hevea brasiliensis) SEEDLINGS IN DIFFERENT TYPES OF SUBSTRATE AND GROW BAG}

\begin{abstract}
The rubber tree (Hevea brasiliensis) is native to the Amazon basin, and its latex is used to produce rubber for the manufacture of tires and other industrialized items. This species is a robust tree that can adapt to the conditions found in a number of different regions of Brazil. One of the most important considerations for the establishment of a commercial rubber plantations is the quality of the growing stock, with the size of the grow bag in which the seed is planted and the type of substrate being the principal factors determining the development of the seedling. The present study investigated the development of rubber tree seedlings planted in different substrates and grow bags of varying sizes. Four
\end{abstract}


types of substrate were tested: standard commercial Carolina substrate (control), bovine manure + sugarcane bagasse (1:1), humus + sugarcane bagasse (1:1), and humus. Polyethylene grow bags of three different sizes were also tested: $25 \mathrm{~cm}$ x $35 \mathrm{~cm}, 20 \mathrm{~cm}$ x 30 $\mathrm{cm}$, and $18 \mathrm{~cm} \times 32 \mathrm{~cm}$. The experimental design was based on a randomized $3 \times 4$ factorial layout, with three grow bag sizes and four types of substrate, and one rubber tree clone, with five replicates. The results of the study indicated that the commercial substrate produced the best initial development of the rubber tree seedlings, under the experimental conditions tested. This substrate, combined with the medium-sized grow bags $(20 \mathrm{~cm} \times 30 \mathrm{~cm})$, guaranteed the best development of the roots, contributing to the establishment of the seedlings under nursery conditions.

Key words: Development. Hevea brasiliensis. Propagation.

\section{INTRODUÇÃO}

A seringueira (Hevea brasiliensis Muell. Arg.) é uma espécie nativa da Amazônia, conhecida popularmente como árvore da borracha natural (APABOR, 2011). O Brasil responde apenas por $35 \%$ do total de borracha natural requerido pelas indústrias nacionais, os outros 65\% são importados da Tailândia, Malásia e Vietnã, e dentre os maiores produtores nacionais destacam-se os estados de São Paulo (54\%), Bahia (13,8\%) e Mato Grosso $(13,5 \%)$ (BASTOS, 2014).

O Estado de Mato Grosso tem como principais atividades econômicas a produção de grãos e a pecuária extensiva (CONAB, 2016). Entretanto, o cultivo da seringueira no estado tem ganhado importância (SOMAIN e DROULERS, 2017) devido às condições edafoclimáticas desfavoráveis ao desenvolvimento do fungo Microcyclus ulei (P. Henn.) v. Arx, causador do mal-das- folhas, o qual tem dificultado a permanência e a expansão da heveicultura na região amazônica (PEREIRA, 1997).

Silva et al. (2001) relataram que o sucesso na implantação de uma cultura depende da qualidade genética, fisiológica e sanitária da muda. Neste sentido, é importante ressaltar que dentre os principais fatores que proporcionam um desenvolvimento satisfatório das mudas está o substrato (PEIXOTO, 1986). O substrato é definido como um meio que possibilita às plantas sustentação e disponibiliza em quantidades suficientes água, oxigênio e nutrientes, além de oferecer $\mathrm{pH}$ e condutividade elétrica de acordo com a necessidade da planta (GUERRINI e TRIGUEIRO, 2004).

Além de proporcionar boas características físicas e químicas, que favoreçam um bom crescimento das mudas, o substrato deve ser abundante na região e gerar baixo custo ao produtor (MELO, 2008). Por outro lado, o aproveitamento de resíduos das atividades agropecuárias e agroindustriais na composição de substratos reduz os impactos ambientais, além dos custos de produção (BEZERRA et al., 2009).

Segundo Brito et al. (2017) podem ser utilizados diversos materiais para a produção de substratos, a partir de uma ou mais texturas, entretanto, esses autores ressaltam a 
importância de que esses sejam isentos de patógenos e que a empresa fabricante possua registro no Ministério da Agricultura, Pecuária e Abastecimento - MAPA.

Aliado a um bom substrato é importante escolher de forma criteriosa as dimensões dos recipientes, pois estes podem interferir diretamente no crescimento da muda (FURTADO, 2007). Melo (2008) define recipiente como material voltado para acondicionar o substrato durante a produção de mudas. Um recipiente considerado adequado deve ser resistente para suportar a pressão exercida pelo substrato, possibilitar um rápido crescimento inicial da muda, possuir bom sistema de drenagem, apresentar durabilidade, baixo custo e ser de fácil manejo (KITAMURA, 2002).

Para Gomes et al. (1990) as pesquisas referentes as dimensões dos recipientes e os tipos de substratos são de suma importância para a produção de mudas de espécies que possuem o desenvolvimento mais lento ou que necessitam permanecerem por mais tempo no viveiro, como é o caso da seringueira (Hevea brasiliensis). Diante do exposto, este trabalho tem como objetivo avaliar o desenvolvimento de mudas de seringueira cultivadas em diferentes substratos e recipientes, em Nova Xavantina-MT.

\section{MATERIAL E MÉTODOS}

O experimento foi conduzido no período de Março a Julho de 2014 no viveiro de mudas da Universidade do Estado de Mato Grosso, Campus de Nova Xavantina-MT, localizado nas coordenadas $14^{\circ} 41^{\prime} 25^{\prime}$ 'S e $52^{\circ} 20^{\prime} 55^{\prime}$ 'W e altitude de $275 \mathrm{~m}$.

O clima da região é do tipo Aw na classificação de Köppen, com quatro meses de seca, de maio a agosto, com precipitação anual de $1.750 \mathrm{~mm}$ e temperatura média mensal de $28^{\circ} \mathrm{C}$ (PORTAL, 2014). A cobertura vegetal da região caracteriza-se pela ocorrência de duas zonas ecológicas, sendo o domínio dos Cerrados e das Florestas de Transição da Pré-Amazônia (MARIMON et al., 2010).

Os substratos utilizados foram Húmus (T1); Bagaço de cana + esterco bovino (1:1) (T2); Carolina Padrão comercial (testemunha) (T3) e Bagaço de cana + húmus (1:1) (T4). Os substratos não comerciais foram homogeneizados e passados em peneira de 7,93 $\mathrm{mm}$, antes de serem submetidos às análises físicas de densidade aparente, porosidade total, granulométrica capacidade de retenção de água, e em peneiras de $2 \mathrm{~mm}$ para análises químicas quanto aos teores de macro e micronutrientes, $\mathrm{pH}$, condutividade elétrica e CTC, conforme metodologia utilizada por Santos et al. (2002). As análises químicas e físicas dos substratos foram realizadas no Laboratório Plante Certo, em Várzea Grande-MT e os resultados das análises estão descritos na Figura 1.

Com o objetivo de formar mudas para produção de porta-enxertos, as sementes utilizadas no experimento foram coletadas de clones GT1, durante o mês de fevereiro. A coleta foi realizada em seringais instalados no Sítio VM3, localizado no município de Nova Xavantina-MT. A semeadura foi realizada manualmente, em sementeiras de $1 \mathrm{~m}^{2}$, sendo utilizado como substrato areia grossa em camadas de $20 \mathrm{~cm}$. Após 20 dias, quando as plântulas alcançaram $10 \mathrm{~cm}$ de altura e estavam no estádio de "palito", foi feita uma seleção, onde foram escolhidas as plântulas que apresentavam parte aérea e sistema radicular normal. 
Após a seleção, as plântulas foram transplantadas para sacos plásticos de polietileno nas dimensões de $25 \times 35 \mathrm{~cm}, 18$ x $32 \mathrm{~cm}$ e 20 x $30 \mathrm{~cm}$, contendo os substratos previamente umedecidos. Após o enchimento dos sacos plásticos, os mesmos foram dispostos em bancadas para serem avaliados.

\begin{tabular}{|c|c|c|c|c|c|c|c|c|c|c|c|}
\hline \multirow[b]{2}{*}{ AMOSTRA } & \multicolumn{6}{|c|}{ MACRONUTRIENTES (g/Kg) } & \multicolumn{5}{|c|}{ MICRONUTRIENTES (mg/Kg) } \\
\hline & $\begin{array}{c}\mathbf{N} \\
\text { (Total) }\end{array}$ & $\begin{array}{c}\mathrm{P}_{2} \mathrm{O}_{5} \\
\text { (Total) }\end{array}$ & $\begin{array}{c}\mathbf{K}_{2} \mathrm{O} \\
\text { (Total) }\end{array}$ & Ca & Mg & $\mathrm{s}$ & Zn & $\mathrm{Cu}$ & $\mathrm{Fe}$ & Mn & B \\
\hline Húmus & 5,5 & 4,4 & 4,1 & 11,5 & 74,5 & 3,3 & 38,9 & 20,8 & 26480,0 & 197,6 & 34,0 \\
\hline Bagaço cana+esterco & 11,7 & 8,7 & 8,4 & 10,7 & 5,1 & 2,4 & 122,3 & 29,9 & 16905,0 & 388,0 & 40,4 \\
\hline Substrato comercial & 9,6 & 6,4 & 4,3 & 6,4 & 3,6 & 1,4 & 62,1 & 16,7 & 6841,0 & 296,7 & 21,0 \\
\hline Bagaço cana+húmus & 16,6 & 10,1 & 8,4 & 9,8 & 4,7 & 3,0 & 149,7 & 33,0 & 33540,0 & 522,9 & 54,9 \\
\hline \multirow{2}{*}{ AMOSTRA } & \multirow{2}{*}{$\begin{array}{c}\text { MO } \\
(\mathrm{g} / \mathrm{kg})\end{array}$} & \multirow{2}{*}{$\begin{array}{c}\mathrm{C} . \\
\text { orgânico } \\
\text { Total } \\
(\mathrm{g} / \mathrm{kg})\end{array}$} & \multirow{2}{*}{$\begin{array}{c}\text { Relação } \\
\mathbf{C} / \mathbf{N}\end{array}$} & \multirow{2}{*}{$\mathrm{pH}$} & \multirow{2}{*}{$\begin{array}{c}\text { Umidade } \\
65^{\circ} \mathrm{C} \\
(\%)\end{array}$} & \multirow{2}{*}{$\begin{array}{l}\text { Condut. } \\
\text { elétrica } \\
(\mu / \mathrm{cm})\end{array}$} & \multirow{2}{*}{$\begin{array}{c}\text { Densidade } \\
\left(\mathrm{kg} / \mathrm{m}^{3}\right)\end{array}$} & \multicolumn{2}{|c|}{$\begin{array}{l}\text { Pen eira (\%) } \\
\text { ABNT N } 40\end{array}$} & \multicolumn{2}{|c|}{$\begin{array}{l}\text { Peneira (\%) } \\
\text { ABNT No4 }\end{array}$} \\
\hline & & & & & & & & $\begin{array}{c}\text { Retido } \\
(\%)\end{array}$ & $\begin{array}{c}\text { Passado } \\
(\%)\end{array}$ & $\begin{array}{c}\text { Retido } \\
(\%)\end{array}$ & Passado (\%) \\
\hline Húmus & 470,0 & 261,2 & 47,5 & 6,0 & 49,5 & 272,8 & 146,3 & 0,3 & 99,7 & 51,4 & 48,4 \\
\hline Bagaço cana+esterco & 342,9 & 190,5 & 16,3 & 7,3 & 42,6 & 800,9 & 366,6 & 2,5 & 97,5 & 43,0 & 54,5 \\
\hline Substrato comercial & 290,2 & 161,3 & 16,8 & 7,4 & 48,1 & 680,9 & 320,4 & 10,3 & 89,7 & 36,0 & 53,7 \\
\hline Bagaço cana+húmus & 325,1 & 180,6 & 10,9 & 6,3 & 44,3 & 626,1 & 445,4 & 0,0 & 100,0 & 36,1 & 63,9 \\
\hline
\end{tabular}

Figura 1. Resultados da análise química dos substratos utilizados no experimento. Fonte: Laboratório Plante Certo (2014). Results of the substrates chemical analyses used in the experiment. Source: Laboratory Plante Certo, Brazil (2014).

O controle fitossanitário, principalmente de doenças fúngicas foi realizado com produtos a base de cobre. As mudas foram mantidas em ambiente protegido sob sombrite de $50 \%$ (Figura 2) e com irrigação diária, com auxílio de regador e aspersor, duas vezes ao dia, devido problemas que ocorreram durante o manuseio de irrigação das mudas, foi utilizado também o regador.

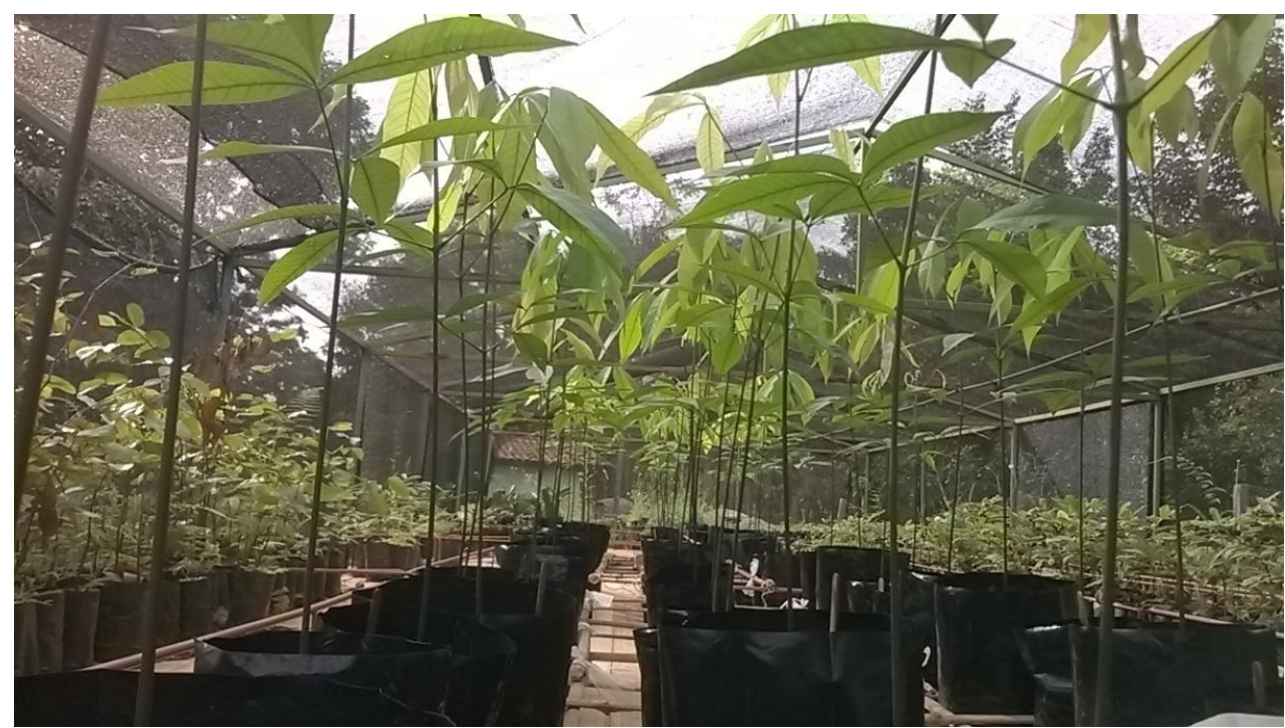

Figura 2. Mudas de seringueira conduzidas no viveiro- Nova Xavantina-MT. Rubber tree seedlings conducted in the nursery-Nova Xavantina-MT.

Aos 90 dias após o transplantio as mudas foram avaliadas quanto a: altura de planta AP (cm) utilizando uma trena graduada; diâmetro do caule - DC (mm) com auxílio de um paquímetro analógico; número de trifólios - NT; comprimento de raiz - $\mathrm{CR}(\mathrm{cm})$; relação altura e diâmetro (H/D); massa seca foliar - MSF (g); massa seca de raiz - MSR (g); massa 
seca do caule - MSC (g); massa seca total - MST (g). Para obtenção da massa seca de cada órgão das mudas, os mesmos foram acondicionados em sacos de papel e levados a estufa de circulação a $60^{\circ} \mathrm{C}$ até atingirem peso constante, e em seguida foram pesados em balança digital com quatro casas decimais.

O delineamento experimental utilizado foi o de blocos casualizados (DBC) em esquema fatorial 3x4, sendo os fatores analisados três recipientes (sacos plásticos com diferentes dimensões), quatro tipos de substratos, totalizando 12 tratamentos, com cinco repetições.

Os dados foram tabulados e as médias comparadas pelo teste de Tukey a 5\% de probabilidade, com auxílio do programa ASSISTAT 7.7 Beta (SILVA e AZEVEDO, 2016).

\section{RESULTADOS E DISCUSSÃO}

A Tabela 1 apresenta o resumo da análise de variância para os parâmetros altura de plantas (AP), diâmetro de caule (DC), número de trifólios (NT), comprimento de radícula $(\mathrm{CR})$ e a relação altura e diâmetro (H/D). Houve diferença significativa pelo teste $\mathrm{F}$ a $1 \%$ de probabilidade do fator substrato para as variáveis AP, DC, NT e H/D, com coeficiente de variação de 14,$69 ; 7,41 ; 37,04$ e 30,77\% respectivamente. Relativamente alto para NT, o que pode ser explicado pela variação no número de folhas de uma planta para outra em função dos tratamentos utilizados.

Para o fator recipiente houve efeito significativo ao nível de $1 \%$ de probabilidade ( $\mathrm{p}<$ 0,01) pelo teste $\mathrm{F}$ somente para $\mathrm{CR}$ e $\mathrm{DC}$, os demais parâmetros não apresentaram diferenças entre si. Para a fonte de variação tratamentos houve diferença significativa $(\mathrm{p}<0,01)$ para todas as variáveis analisadas neste experimento. $\mathrm{Na}$ interação substrato $\mathrm{x}$ recipiente $\mathrm{o} \mathrm{CR}$ apresentou diferença significativa ao nível de 5\% de probabilidade pelo teste $\mathrm{F}$.

A Tabela 2 apresenta o resumo da análise da variância para os parâmetros massa seca foliar (MSF), massa seca de raiz (MSR), massa seca do caule (MSC), massa seca total (MST), Para o tratamento substrato houve efeito significativo ao nível de $1 \%$ de probabilidade para todos os parâmetros avaliados, apresentando coeficientes de variação de 28,$67 ; 27,95 ; 22,49$ e 21,21, respectivamente. Em relação ao recipiente a MSF apresentou diferença significativa ao nível de $1 \%(\mathrm{p}<0,01)$ e MST a $5 \%$ de probabilidade pelo teste F. Não houve efeito significativo entre a interação substrato $\mathrm{x}$ recipiente nas variáveis avaliadas neste experimento.

Em geral o substrato 3 (substrato comercial) apresentou melhores resultados para a maioria dos parâmetros avaliados: DC (0,99 mm), CR (27,87 cm), MSR (5,44 g), MSC (7,01 g), MST (17,06 g) (Tabela 3). Para NT (10,53 cm) e MSF (4,61 g) os valores foram inferiores ao substrato 4 (bagaço de cana + húmus) que obteve numericamente valores superiores $(\mathrm{NT}=$ $12,53 \mathrm{~cm}$ e MSF = 4,70 g) para esses parâmetros, embora não tenham diferido estatisticamente entre si (Tabela 3). 
Tabela 1. Resumo da análise de variância (ANOVA) para os parâmetros altura de plantas (AP), diâmetro do caule (DC), número de folíolos (NF), comprimento de raiz (CR) e relação altura e diâmetro (H/D) de mudas de seringueira submetidas a diferentes substratos e recipientes. Nova Xavantina - MT. Summary of variance analysis (ANOVA) for plant height $(A P)$, stem diameter $(D C)$, number leaf $(N F)$, root length $(C R)$ and height and diameter ratio $(H / D)$ of rubber tree seedlings submitted to different substrates and containers. Nova Xavantina-MT.

\section{QUADRADO MÉDIO}

\begin{tabular}{lcccccc}
\cline { 5 - 7 } \multicolumn{1}{c}{ FV } & GL & AP & DC & NF & CR & H/D \\
\hline Substrato & 3 & $18,87^{* *}$ & $59,29^{* *}$ & $10,93^{* *}$ & $0,98^{\text {ns }}$ & $29,98^{* *}$ \\
Recipiente & 2 & $1,47^{\text {ns }}$ & $5,43^{* *}$ & $0,21^{\text {ns }}$ & $5,79^{* *}$ & $0,77^{\text {ns }}$ \\
Substrato x Recipiente & 6 & $1,72^{\text {ns }}$ & $1,15^{\text {ns }}$ & $0,78^{\text {ns }}$ & $2,94^{*}$ & $0,48^{\text {ns }}$ \\
\hline Tratamentos & 11 & $6,35^{* *}$ & $17,79^{* *}$ & $3,44^{* *}$ & $2,93^{* *}$ & $8,58^{* *}$ \\
Blocos & 4 & $0,49^{\text {ns }}$ & $0,79^{\text {ns }}$ & $0,51^{\text {ns }}$ & $1,37^{\text {ns }}$ & $0,62^{\text {ns }}$ \\
Resíduo & 44 & ----- & ----- & ----- & ----- & ----- \\
\hline CV\% & ----- & 14,69 & 7,41 & 37,04 & 18,94 & 30,77 \\
\hline
\end{tabular}

** significativo ao nível de $1 \%$ de probabilidade $(\mathrm{p}<0,01)$ pelo teste $\mathrm{F}$; *significativo ao nível de $5 \%$ de probabilidade $(0,01 \leq \mathrm{p}<0,05)$; ns - não significativo $(\mathrm{p} \geq 0,05)$. CV - coeficiente de variação. ** significant at the $1 \%$ probability level $(p<0.01)$ by the $F$ test; * significant at the $5 \%$ probability level $(0.01 \leq p<0.05)$; $n s$ not significant $(p \geq 0.05)$. $C V$ - coefficient of variation.

Tabela 2. Resumo da análise de variância (ANOVA) para os parâmetros massa seca foliar (MSF), massa seca de raiz (MSR), massa seca do caule (MSC), massa seca total (MST) de mudas de seringueira submetidas a diferentes substratos e recipientes. Nova Xavantina - MT. Summary of variance analysis (ANOVA) for leaf dry matter (MSF), roots dry matter (MSR), stem dry matter (MSC), total dry matter (MST) of rubber tree seedlings submitted to different substrates and containers. Nova Xavantina - MT.

\begin{tabular}{lccccc}
\hline & & \multicolumn{4}{c}{ Quadrado Médio } \\
\cline { 3 - 6 } \multicolumn{1}{c}{ FV } & GL & MSF & MSR & MSC & MST \\
\hline Substrato & 1 & $59,56^{* *}$ & $48,57^{* *}$ & $63,77^{* *}$ & $79,76^{* *}$ \\
Recipiente & 2 & $5,73^{* *}$ & $0,89^{\text {ns }}$ & $2,51 \mathrm{~ns}$ & $3,67^{*}$ \\
Substrato x Recipiente & 6 & $1,04^{\text {ns }}$ & $1,77^{\text {ns }}$ & $1,04 \mathrm{~ns}$ & $1,29^{\text {ns }}$ \\
\hline Tratamentos & 11 & $17,85^{* *}$ & $14,37^{* *}$ & $18,42^{* *}$ & $23,12^{* *}$ \\
Blocos & 4 & $1,66^{\text {ns }}$ & $1,09^{\text {ns }}$ & $0,73^{\text {ns }}$ & $1,27^{\text {ns }}$ \\
Resíduo & 44 & ----- & ----- & ---- & ---- \\
\hline CV\% & ----- & 28,67 & 27,95 & 22,49 & 21,21 \\
\hline
\end{tabular}

** significativo ao nível de $1 \%$ de probabilidade $(\mathrm{p}<0,01)$ pelo teste $\mathrm{F}$; *significativo ao nível de $5 \%$ de probabilidade $(0,01 \leq \mathrm{p}<0,05)$; ns - não significativo $(\mathrm{p} \geq 0,05)$. CV - coeficiente de variação. ** significant at the $1 \%$ probability level $(p<0.01)$ by the F test; * significant at the $5 \%$ probability level $(0.01 \leq p<0.05)$; $n s$ not significant $(p \geq 0.05)$. $C V$ - coefficient of variation.

Para altura de plantas o substrato 4 (bagaço de cana + húmus) proporcionou maiores resultados $(80,73 \mathrm{~cm})$, seguido do substrato comercial $(70,47 \mathrm{~cm})$ e bagaço de cana + esterco bovino $(63,80 \mathrm{~cm})$ e o substrato 1 (húmus) o menor valor $(54,4 \mathrm{~cm})$. Já para DC o substrato comercial obteve o maior valor $(0,99 \mathrm{~mm})$ quando comparado ao húmus. 
Tabela 3. Valores médios para altura de plantas (AP), diâmetro do caule (DC), número de folíolos (NF), comprimento de radícula (CR), relação altura e diâmetro (HD), massa seca foliar (MSF), massa seca de raiz (MSR), massa seca do caule (MSC), massa seca total (MST) de mudas de seringueira submetidas a diferentes substratos e recipientes. Nova Xavantina MT. Average values for plant height (AP), stem diameter $(D C)$, leaf number $(N F)$, root length (CR), height and diameter ratio (HD), leaf dry matter (MSF), root dry matter (MSR), stem dry matter (MSC), total dry matter (MST) of rubber tree seedlings submitted to different substrates and containers. Nova Xavantina - MT.

\begin{tabular}{c|ccccccccc}
\hline Substrato & $\begin{array}{c}\text { AP } \\
(\mathbf{c m})\end{array}$ & $\begin{array}{c}\text { DC } \\
(\mathbf{m m})\end{array}$ & $\mathbf{N F}$ & $\begin{array}{c}\text { CR } \\
(\mathbf{c m})\end{array}$ & $\begin{array}{c}\text { H/D } \\
\mathbf{M S F}\end{array}$ & $\begin{array}{c}\text { MSR } \\
(\mathbf{g})\end{array}$ & $\begin{array}{c}\text { MSC } \\
(\mathbf{g})\end{array}$ & $\begin{array}{c}\text { MST } \\
(\mathbf{g})\end{array}$ \\
\hline $\mathbf{1}$ & $54,4 \mathrm{c}$ & $0,69 \mathrm{c}$ & $6,33 \mathrm{~b}$ & $29,53 \mathrm{a}$ & $79,53 \mathrm{a}$ & $1,18 \mathrm{~b}$ & $1,46 \mathrm{c}$ & $2,32 \mathrm{~d}$ & $4,96 \mathrm{~d}$ \\
$\mathbf{2}$ & $63,80 \mathrm{bc}$ & $0,83 \mathrm{~b}$ & $7,20 \mathrm{~b}$ & $26,77 \mathrm{a}$ & $76,94 \mathrm{a}$ & $2,05 \mathrm{~b}$ & $2,91 \mathrm{~b}$ & $3,37 \mathrm{c}$ & $8,32 \mathrm{c}$ \\
$\mathbf{3}$ & $70,47 \mathrm{~b}$ & $0,99 \mathrm{a}$ & $10,53 \mathrm{a}$ & $27,87 \mathrm{a}$ & $23,55 \mathrm{~b}$ & $4,61 \mathrm{a}$ & $5,44 \mathrm{a}$ & $7,01 \mathrm{a}$ & $17,06 \mathrm{a}$ \\
$\mathbf{4}$ & $80,73 \mathrm{a}$ & $0,87 \mathrm{~b}$ & $12,53 \mathrm{a}$ & $26,63 \mathrm{a}$ & $86,35 \mathrm{a}$ & $4,70 \mathrm{a}$ & $3,28 \mathrm{~b}$ & $4,87 \mathrm{~b}$ & $12,84 \mathrm{~b}$ \\
\hline Dms & 9,64 & 0,072 & 3,3 & 5,11 & 19,98 & 0,88 & 0,89 & 0,96 & 2,23 \\
\hline Recipiente & $\mathbf{A P}$ & $\mathbf{D C}$ & $\mathbf{N T}$ & $\mathbf{C R}$ & $\mathbf{H} / \mathbf{D}$ & $\mathbf{M S F}$ & MSR & MSC & MST \\
\hline $\mathbf{1}$ & $66,7 \mathrm{a}$ & $0,85 \mathrm{ab}$ & $8,75 \mathrm{a}$ & $30,85 \mathrm{a}$ & $69,95 \mathrm{a}$ & $2,74 \mathrm{~b}$ & $3,24 \mathrm{a}$ & $4,28 \mathrm{a}$ & $10,26 \mathrm{a}$ \\
$\mathbf{2}$ & $65,05 \mathrm{a}$ & $0,81 \mathrm{~b}$ & $9,40 \mathrm{a}$ & $25,40 \mathrm{~b}$ & $67,67 \mathrm{a}$ & $3,00 \mathrm{ab}$ & $3,09 \mathrm{a}$ & $4,11 \mathrm{a}$ & $10,21 \mathrm{a}$ \\
$\mathbf{3}$ & $70,3 \mathrm{a}$ & $0,88 \mathrm{a}$ & $9,30 \mathrm{a}$ & $26,85 \mathrm{ab}$ & $62,14 \mathrm{a}$ & $3,67 \mathrm{a}$ & $3,48 \mathrm{a}$ & $4,78 \mathrm{a}$ & $11,93 \mathrm{a}$ \\
\hline Dms & 7,58 & 0,061 & 2,59 & 4,02 & 15,71 & 0,69 & 0,7 & 0,76 & 1,76 \\
\hline
\end{tabular}

Médias seguidas de mesma letra não diferem entre si pelo teste de Tukey a 5\% de probabilidade. Substratos: 1 (Húmus); 2 (Bagaço de cana + esterco bovino); 3 (Substrato comercial); 4 (Bagaço de cana + húmus). Recipientes: $1(25 \times 35 \mathrm{~cm}) ; 2(18 \times 32 \mathrm{~cm}) ; 3(20 \times 30 \mathrm{~cm})$. Means followed by the same letter do not differ by Tukey test at 5\% probability. Substrates: 1 (Humus); 2 (cane bagasse + bovine manure); 3 (Commercial Substrate); 4 (Sugarcane bagasse + humus). Containers: $1(25 \times 35 \mathrm{~cm}) ; 2(18 \times 32 \mathrm{~cm}) ; 3(20 \times 30 \mathrm{~cm})$.

Danner et al. (2007) relataram que, em geral, o diâmetro do colo é analisado para indicar a capacidade de sobrevivência da muda no campo, além de ser parâmetro para a definição de doses de fertilizantes a serem aplicadas na produção de mudas. Mudas com baixo diâmetro de colo apresentam dificuldades de se manterem eretas após o plantio. Neste caso, a utilização do substrato comercial obteve resultados satisfatórios nesse parâmetro.

Para a relação altura e diâmetro do caule (H/D) os tratamentos 1, 2 e 4 apresentaram maiores valores $(79,53 ; 76,94$ e 86,35, respectivamente), não diferindo estatisticamente entre si e o substrato comercial o menor valor $(23,55)$. De acordo com Sturion (2000), a relação altura/diâmetro do colo constitui um dos parâmetros usados para se avaliar a qualidade de mudas florestais, pois, além de refletir o acúmulo de reservas, assegura maior resistência e melhor fixação no solo, quanto menor a relação a possibilidade de estabelecimento a campo são maiores.

Os menores resultados para os parâmetros avaliados foram apresentados pelos substratos 1 (húmus) CE:272,8 u/cm e 2 (Bagaço de cana + esterco bovino) CE:800,9 u/cm, respectivamente, com DC (0,69 e 0,83 cm), NT (6,33 e 7,20), MSF (1,18 e 2,08 g), MSR (1,46 e 2,91 g), MSC (2,32 e 3,37 g), MST (4,96 e 8,32 g).

Para o fator recipiente, em média os maiores valores para $\mathrm{CR}$ foram encontrados quando se utilizou o recipiente com dimensões $(25 \times 35 \mathrm{~cm})$, onde pode notar visualmente o 
desenvolvimento das raízes de maneira mais uniforme que nos outros dois recipientes. $\mathrm{O}$ menor valor $(25,40 \mathrm{~cm})$ foi encontrado para o recipiente $2(18 \times 32 \mathrm{~cm})$. Em termos de MSF o recipiente 3 apresentou maior valor $(3,67 \mathrm{~g})$ e o recipiente 1 o menor valor $(2,74 \mathrm{~g})$. Nos demais parâmetros não houve diferença estatística entre as médias dos tratamentos utilizados.

Colaborando com os resultados de Sturion (2000) que comprovaram que as mudas de seringueira cultivadas em recipientes com maior volume apresentaram maior diâmetro de colo, em relação aquelas cultivadas em recipientes menores. O mesmo foi observado por Cunha et al. (2005) em que recipientes de maiores volumes oferecem melhores condições para o desenvolvimento das mudas, mas eles somente devem ser utilizados para espécies que apresentam desenvolvimento lento, necessitando permanecer no viveiro por um longo tempo, entre 10 a 12 meses ou quando se desejam mudas bem desenvolvidas, como é o caso da seringueira.

Já Barroso (2000) encontraram resultados diferentes, trabalhando com mudas de espécies florestais onde em recipientes muito altos, a disponibilidade de oxigênio na parte inferior fica reduzida se o substrato não for bem arejado, o que prejudica a respiração e o crescimento radicular e pode propiciar o desenvolvimento de doenças, recomendando que a altura do recipiente seja no máximo $22 \mathrm{~cm}$ para que não haja problemas de aeração.

Pereira (1985) analisando o tamanho do recipiente para a produção de mudas de seringueira na Amazônia constataram que recipientes de 15 x $35 \mathrm{~cm}$ ou 15 x $40 \mathrm{~cm}$ são tão eficientes quanto os normalmente utilizados $(56 \times 25 \mathrm{~cm})$, apresentando a vantagem adicional de reduzirem os custos de produção, transporte e plantio em cerca de $60 \%$.

Tabela 4. Desdobramento da interação substrato x recipiente para o parâmetro comprimento de raiz $(\mathrm{CR})$ de mudas de seringueira submetidas a diferentes substratos e recipientes. Nova Xavantina - MT. Split substrate interaction $x$ container of the root length $(C R)$ of rubber tree seedlings submitted to different substrates and containers. Nova Xavantina - MT.

\begin{tabular}{l|ccc}
\hline \multirow{2}{*}{\multicolumn{1}{c|}{ Substratos }} & \multicolumn{3}{c}{ Recipientes } \\
\cline { 2 - 4 } & $\mathbf{2 5 \times 3 5} \mathbf{3 5 m}$ & $\mathbf{1 8} \mathbf{3 2} \mathbf{~ c m}$ & $\mathbf{2 0} \mathbf{3 0} \mathbf{~ c m}$ \\
\hline Húmus & $32,40 \mathrm{aA}$ & $27,20 \mathrm{a} \mathrm{A}$ & $29,00 \mathrm{abA}$ \\
Bagaço de cana + Esterco bovino & $31,50 \mathrm{aA}$ & $26,40 \mathrm{aA}$ & $22,40 \mathrm{bB}$ \\
Substrato comercial & $25,60 \mathrm{aA}$ & $26,20 \mathrm{aA}$ & $31,80 \mathrm{aA}$ \\
Bagaço de cana + Húmus & $33,90 \mathrm{aA}$ & $21,80 \mathrm{aB}$ & $24,20 \mathrm{abB}$ \\
\hline
\end{tabular}

Médias seguidas de mesma letra minúscula nas colunas e maiúscula nas linhas não diferem entre si pelo teste de Tukey a 5\% de probabilidade. DMS para colunas = 8,86 e DMS para linhas =8,05. Means followed by the same lowercase letter in the columns and upper case in the lines do not differ from each other by the Tukey test at 5\% probability. DMS for columns $=8.86$ and DMS for rows $=8.05$.

A Tabela 4 apresenta o desdobramento da interação substrato x recipiente para o CR. Quando se analisa o substrato dentro do fator recipiente, em média os maiores valores de CR foram encontrados quando combinou bagaço de cana + esterco bovino com os recipientes 1 $(25 \times 35 \mathrm{~cm})$ e $2(18 \times 32 \mathrm{~cm})$, embora não tenham diferido entre si pelo teste de Tukey a $5 \%$ de probabilidade. Para o substrato bagaço de cana + húmus combinado ao recipientes 1 ( $25 \mathrm{x}$ $35 \mathrm{~cm}$ ). E os menores valores para a combinação dos mesmos substratos mencionados com o recipiente $3(20 \times 30 \mathrm{~cm})$. Já quando analisamos o fator recipiente dentro de substrato o 
maior valor de CR $(31,80 \mathrm{~cm})$ foi encontrado quando se utilizou o recipiente $3(20 \times 30 \mathrm{~cm})$ combinado ao substrato comercial e o menor valor $(22,40 \mathrm{~cm})$ quando o mesmo recipiente continha bagaço de cana + esterco bovino.

Resultados semelhantes foram encontrados por Carvalho Filho et al. (2004), estudando a produção de mudas de angelim em diferentes ambientes, recipientes e substratos, verificaram que houve interação entre os fatores recipiente e substrato, sendo o recipiente maior e o substrato contendo solo + esterco (2:1) e/ou solo + areia + esterco $(1: 2: 1)$ responsáveis pelo melhor desenvolvimento das mudas. Assim como Viana et al. (2008) analisando o crescimento de mudas de Bauhinia forficata (Pata de Vaca) concluíram que o recipiente com maior volume de substrato proporcionou os maiores valores e o menor resultado foi encontrado quando as mudas estavam no menor recipiente.

\section{CONCLUSÃO}

O substrato comercial é o mais indicado para o desenvolvimento inicial de mudas de seringueira, nas condições do experimento. O mesmo combinado ao recipiente (saco de polietileno tamanho $20 \times 30 \mathrm{~cm}$ ) permitiu um maior comprimento de raízes, influenciando o estabelecimento de plântulas em condições de viveiro.

\section{REFERÊNCIAS BIBLIOGRÁFICAS}

ASSOCIAÇÃO PAULISTA DE PRODUTORES E BENIFICIADORES DE BORRACHA APABOR. Heveicultura. Informativo Apabor, São José do Rio Preto, v. 4, n. 1, p.1-8, 2011.

BARROSO, D. G. Efeitos do recipiente sobre o desempenho pós-plantio de Eucalyptus camaldulensis Dehnh. e E. urophylla S. T. Blake. Revista Árvore, Viçosa, v. 24, n. 3, p.291296, 2000.

BASTOS, T. R. Brasil produz apenas $35 \%$ da demanda nacional por borracha natural. Disponível em: <http://revistagloborural.globo.com/Noticias/Agricultura/noticia/2014/06/brasil-produzapenas-35-da-demanda-nacional-por-borracha-natural.html>. Acesso em: 02 nov. 2014.

BEZERRA, F. C.; FERREIRA, F. V. M.; SILVA, T. C. Produção de mudas de berinjela em substratos à base de resíduos orgânicos e irrigadas com água ou solução nutritiva. Horticultura Brasileira, Fortaleza, v. 27, n. 2, p.1348-1352, 2009. Disponível em: <http://www.abhorticultura.com.br/eventosx/trabalhos/ev_3/a1962_t2939_comp.pdf >. Acesso em: 05 jan. 2018.

BRITO, P. F.; MARTINS, A. L. M.; LUCA, C. A.; GONÇALVES, E. C. P. Produção de mudas de seringueira em bancada e substrato (viveiro suspenso). Disponível em: <https://www.defesa.agricultura.sp.gov.br/arquivos/sanidade-vegetal/seringueira/ seringueiramanual-legislacao-01-02-2017.pdf>. Acesso em: 21 abr. 2018.

CARVAlHO FILHO, J. L. S.; ARRIGONIBLANK, M. F.; BLANK, A. F. Produção de mudas de angelim (Andira fraxinifolia Benth.) em diferentes ambientes, recipientes e 
composições de substratos. Revista Ciência Agronômica, Fortaleza, v. 35, n. 1, p.61-67, 2004. Disponível em: <https://ri.ufs.br/jspui/bitstream/riufs/1624/1/ProducaoMudas Angelim.pdf>. Acesso em: 02 fev. 2018.

COMPANHIA NACIONAL DE ABASTECIMENTO - CONAB. Acompanhamento da safra brasileira de grãos/2016. Disponível em:〈http://www.conab.gov.br $>$. Acesso em: 06 out. 2016.

CUNHA, A. O.; ANDRADE, L. A.; BRUNO, R. L. A.; SILVA, J. A. L.; SOUZA, V. C. Efeitos de substratos e das dimensões dos recipientes na qualidade das mudas de Tabebuia impetiginosa (Mart. Ex D.C.) Standl. Revista Árvore, Viçosa, v. 29, n. 4, p.507-516, 2005. Disponível em: <http://www.redalyc.org/articulo.oa?id=48829402〉. Acesso em 06 jan. 2018.

DANNER, M. A.; CITADIN, L.; FERNANDES JUNIOR, A. A. Formação de mudas de jabuticabeira (Plinia sp.) em diferentes substratos e tamanhos de recipientes. Revista Brasileira de Fruticultura, Jaboticabal, v. 29, n. 1, p.179-182, 2007. Disponível em: <http://www.scielo.br/pdf/rbf/v29n1/a38v29n1.pdf>. Acesso em: 06 jan. 2018.

FURTADO, E. L. Manejo do mal-das-folhas de seringueira no Brasil. Informe Agropecuário, Belo Horizonte, v. 28, n. 237, p.88-94, 2007.

GOMES, J. M.; COUTO, L.; BORGES, R. C. G.; FONSECA, E. P. Efeitos de Diferentes substratos na produção de mudas de Eucalyptus grandis Hill ex Maiden, Win-Strip. Árvore, Viçosa, v. 15, n. 1, p.35-42, 1990.

GUERRINI, I. A.; TRIGUEIRO, R. M. Atributos físicos e químicos de substratos compostos por biossólidos e casca de arroz carbonizada. Revista Brasileira de Ciência do Solo, Viçosa, v. 28, n. 6, p.1069-1076, 2004. Disponível em: <http://www.scielo.br/pdf/rbcs/v28n6/22929.pdf>. Acesso em: 02 fev. 2018.

KITAMURA, M. C. Propagação precoce de gravioleira (AnnonamuricataL.) por enxertia. 2002. 79 f. Tese (Doutorado em Agronomia - Fitotecnia) - Universidade de Lavras, Lavras, 2002.

MARIMON, B. S.; FELFILI, J. M.; LIMA, E. S.; DUARTE, W. M. G.; MARIMONJÚNIOR, B. H. Environmental determinants for natural regeneration of gallery forest at the Cerrado/Amazonia boundaries in Brazil. Acta Amazônica, Manaus, v. 40, n. 1, p.107-118, 2010. Disponível em: <http://www.scielo.br/scielo.php?Script=sci_arttext\&pid=S0044 59672010000100014>. Acesso em: 02 fev. 2018.

MELO, J. K. H. Avaliação de diferentes substratos na produção de porta-enxerto de tamarindeiro (Tamarindus indica L.). 2008. 69 f. Dissertação (Mestrado em Fitotecnia) Universidade Federal Rural do Semi-Árido, Mossoró, 2008.

PEIXOTO, J. R. Efeito da matéria orgânica, do superfosfato simples e do cloreto de potássio na formação de mudas de maracujazeiro azedo (Passiflora edulis f. Flavicarpa DENEGER). 1986. 101 f. Dissertação (Mestrado em Agronomia) - Universidade Federal de Lavras, Lavras, 1986. 
PEREIRA, A. V. Avaliação preliminar do desempenho de clones de seringueira (Hevea spp.) no estado de Goiás e no Distrito Federal. 1997. 98 f. Tese (Doutorado em Agronomia) - Universidade Federal de Lavras, Lavras, 1997.

PEREIRA, A. V; PEREIRA, E. B. C. Influência do tamanho do saco plástico no desenvolvimento de mudas de seringueira, durante a fase de viveiro. Comunicado Técnico, Manaus, v. 23, n. 1, p.8-32, 1985.

PORTAL. Levantamento climático/2014. Disponível em: <www.portalagro.com.br>. Acesso em: 04 nov. 2014.

SANTOS, F. R. P.; SOUZA, L. M.; MARQUES, N. D.; CASTILHO, R. Caracterização físico-química de sete componentes de substratos recomendados para uso em floricultura. Cultura Agronômica, Ilha Solteira, v. 11, n. 2, p.81-92, 2002.

SILVA, F. A. S.; AZEVEDO, C. A. V. The Assistat Software Version 7.7 and its use in the analysis of experimental data. African Journal of Agricultural Research, Uganda, v. 11, n. 39, p.3733-3740, 2016. Disponível em: <http://www.Academicjournals.org/journal/AJAR/article-full-text-pdf/5E8596460818>. Acesso em: 04 jan. 2018.

SILVA, R. P.; PEIXOTO, J. R.; JUNQUEIRA, N. T. V. Influência de diversos substratos no desenvolvimento de mudas de maracujazeiro azedo (Passiflora edulisf. Flavicarpa DENEGER). Revista Brasileira de Fruticultura, Jaboticabal, v. 23, n. 2, p.377-381, 2001. Disponível em: <http://www.scielo.br/scielo.php?pid=S010029452001000200036\&script=sci_abstract\&tlng=pt>. Acesso em: 03 fev. 2018.

SOMAIN, R.; DROULERS, M. A seringueira agora é paulista. Confins, v. 27, n. 1, p.1-6, 2016. Disponível em:< https://journals.openedition.org/confins/10906\#quotation>. Acesso em> 20 out. 2018.

STURION, J. A. Produção de mudas de seringueira. In: SEMINÁRIO SOBRE ATUALIDADES E PERSPECTIVAS FLORESTAIS. Bracatinga uma alternativa para reflorestamento, 4, 1981, Curitiba. Anais... Curitiba: EMBRAPA/URPFCS, 2000. 28 p.

VIANA, J. S.; GONÇALVES, E. P.; ANDRADE, L. A.; OLIVEIRA, L. S. B; SILVA, E. O. Crescimento de mudas de Bauhinia forficata Link. em diferentes tamanhos de recipientes. Floresta, Curitiba, v. 38, n. 4, p.663-671, 2008. Disponível em: <file://C:/Users/user/Downloads/13161-43667-1-PB\%20(1).pdf>. Acesso em: 05 fev.2018. 